\title{
Peramivir, an anti-influenza virus drug, exhibits potential anti-cytokine storm effects
}

$3{ }^{1}$ Institute of Translational Medicine, Shanghai University, Shanghai, China.

$4 \quad{ }^{2}$ Department of Medicine, Shanghai East Hospital, Tongji University, 200120 Shanghai, China

$5 \quad{ }^{3}$ School of Pharmacy, Second Military Medical University, 200433 Shanghai, China

$6 \quad{ }^{4}$ School of Pharmacy, Ningxia Medical University, 750004 Yinchuan, China

7 These authors contributed equally: Chen-xi Zhang(18801759639@163.com) and Ye Tu(dan16065@163.com)

8 Correspondence: Li Su (suli1020@shu.edu.cn), Chun-lin Zhuang (zclnathan@163.com) or Zhi-bin Wang (methyl@smmu.edu.cn)

10 Running title: Peramivir as a potential anti-cytokine storm agent

12 Abstract Coronavirus Disease 2019 (COVID-19) infected by Severe Acute Respiratory Syndrome Coronavirus -2 (SARS-CoV-2) has been 13 declared a public health emergency of international concerns. Cytokine storm syndrome (CSS) is a critical clinical symptom of severe 14 COVID-19 patients, and the macrophage is recognized as the direct host cell of SARS-CoV-2 and potential drivers of CSS. In the present study, 15 peramivir was identified to reduce TNF- $\alpha$ by partly intervention of NF- $\kappa$ B activity in LPS-induced macrophage model. In vivo, peramivir reduced the multi-cytokines in serum and bronchoalveolar lavage fluid (BALF), alleviated the acute lung injury and prolonged the survival time 
17 in mice. In human peripheral blood mononuclear cells (hPBMCs), peramivir could also inhibit the release of TNF- $\alpha$. Collectively, we proposed

18 that peramivir might be a candidate for the treatment of COVID-19 and other infections related CSS.

19

20 KEY WORDS cytokine storm syndrome; COVID-19; peramivir; acute lung injury; multi-cytokines 


\section{Graphic Abstract}

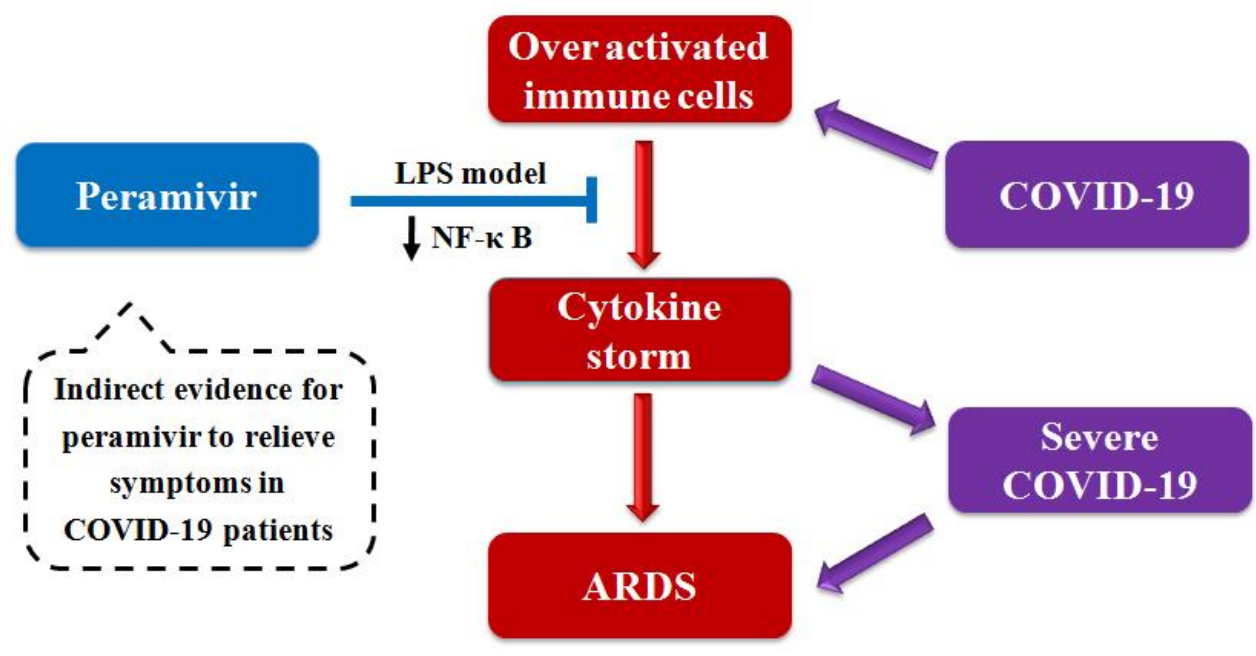




\section{INTRODUCTION}

Coronavirus disease 2019 (COVID-19) caused by Severe Acute Respiratory Syndrome Coronavirus -2 (SARS-CoV-2) has been reported to infect more than 12 million people worldwide killing 500 thousand people (updated on Jul $10^{\text {th }}, 2020$ ). ${ }^{1}$ World Health Organization (WHO) has declared COVID-19 as a public health emergency of international concerns. ${ }^{2}$ COVID-19 patients show typical clinical symptoms of fever, fatigue, dry cough and pneumonia. ${ }^{3-5}$ Excessive inflammatory response leads to acute respiratory distress syndrome (ARDS), coagulopathy, and septic shock which can be fatal in critical cases. ${ }^{3}$ Gross anatomy identifies the main pathological features including exudation and hemorrhage, epithelium injuries, infiltration of inflammatory immune cells and fibrosis in the lungs of fatal patients., 6-10

A syndrome with a distinct cytokine storm was showed in a subgroup of patients with severe COVID-19, which has also been reported in SARS-CoV infected patients. ${ }^{11-14}$ The cytokine storm refers to an uncontrolled excessive inflammatory response, spreading from a local lesion to the whole body through the systemic circulation. ${ }^{15,16}$ The plasma concentrations of inflammation related cytokines such as interleukins (IL) -2 , $-6,-7$, and -10 , tumor necrosis factor- $\alpha$ (TNF- $\alpha$ ), interferon- $\gamma$-inducible protein 10 (IP10), granulocyte-colony stimulating factor (G-CSF), monocyte chemoattractant protein -1 (MCP-1), and macrophage inflammatory protein 1 alpha (MIP-1 $\alpha$ ) were significantly increased in the COVID-19 patients. ${ }^{3,17,18}$ Specially, the activation of alveolar macrophages was a characteristic abnormality. ${ }^{8}$

The leading cause of mortality is thought to be ARDS-induced respiratory failure, and patients generally receive supportive management in clinic practice. ${ }^{17}$ However, there is still no well-accepted effective treatment for COVID-19. The development of novel therapeutics has been mainly focused on antivirals ${ }^{19,20}$ and vaccines. ${ }^{11}$ In addition, the cytokine storm is increasingly being recognized as a key node for the patients deteriorating to severe COVID-19. ${ }^{3,11,17}$ Therefore, anti-inflammatory therapy has been considered as one of appropriate clinical adjuvant 
44 treatment options, and the treatments include steroids (e.g., prednisone), ${ }^{21}$ selective cytokine blockade (e.g., tocilizumab), ${ }^{11} \mathrm{JAK}$ inhibition (e.g.,

45 Baricitinib), ${ }^{22}$ intravenous immunoglobulin, Chinese medicines and blood purification. ${ }^{11,21}$

Early in Feb, 2020, 75 of 99 COVID-19 patients received antiviral treatment including oseltamivir. ${ }^{23}$ Besides, oseltamivir is noted to have been widely used for confirmed or suspected COVID-19 cases in hospitals in China ${ }^{24}$ and Thailand (NCT04303299). The other two clinical trials

48 (NCT04261270 and NCT04255017) involving oseltamivir in the treatment of COVID-19 are currently ongoing. However, the FDA-approved 49 neuraminidase inhibitors including oseltamivir, zanamivir and peramivir were ineffective against the SARS-CoV-2 virus in vitro. ${ }^{25}$ There has 50 been no exact evidence to date that oseltamivir is effective in the treatment of COVID-19 in clinic. ${ }^{19}$

It is reported that oseltamivir exhibited the antiviral activity of reducing pulmonary viral load, thereby the cytokines production was suppressed. ${ }^{26}$ Macrophages play a vital role in both SARS-CoV-2 virus -induced lung lesions and the host cytokine-mediated response. ${ }^{8}$ In our previous study, entecavir, a hepatitis B virus (HBV) inhibitor, was demonstrated to directly inhibit the release of cytokines in lipopolysaccharide (LPS) -stimulated macrophage model, ${ }^{27}$ which is a classic in vitro model to evaluate the anti-inflammatory activity of the drugs. ${ }^{28}$ Herein, we also examined whether these three neuraminidase inhibitors could inhibit the expression of inflammatory cytokines in LPS-stimulated macrophages in the present study. The results showed peramivir had the best ability to inhibit TNF- $\alpha$ by $\sim 70 \%$ among the three compounds.

57 Furthermore, we estimated the anti-cytokine storm effect and lung protection of peramivir in vivo. The anti-inflammatory effect of peramivir in human peripheral blood mononuclear cells (hPBMCs) was also observed.

\section{MATERIALS AND METHODS}




\section{Materials}

62 Compounds were purchased from TargetMol with a purity of > 98\% (TargetMol). LPS (E. coli 0111:B4) was obtained from MilliporeSigma.

\section{Animal}

64 Male C57BL/6J mice (18-22 g) were purchased from the Changzhou Cavens Laboratory Animal Co., China. All mice were kept under an 65 automated $12 \mathrm{~h}$ dark-light cycle at a controlled temperature of $22^{\circ} \mathrm{C} \pm 2{ }^{\circ} \mathrm{C}$ and a relative humidity of $50 \%-60 \%$ with free access to standard dry 66 diet and tap water. All animal experiments were carried out in adherence with the NIH Guide for the Care and Use of Laboratory Animals

67 (National Academies Press, 2011) and were approved by the Second Military Medical University Committee on Animal Care (EC11-055).

\section{CCS model}

69 CCS was induced by a single i.p. injection of LPS ( $15 \mathrm{mg} / \mathrm{kg})$, as described previously. ${ }^{29}$ After $4 \mathrm{~h}$, mice were sacrificed and serum was collected.

70 After $8 \mathrm{~h}$, the left bronchus was ligated and $1 \mathrm{~mL}$ saline was perfused into right lung lobe to collect BALF, and the left lung was fixed with

71 paraformaldehyde for histological analysis. Serum and BALF were further used for multi-cytokine analysis.

72 To investigate the effect of drugs on the survival time of CSS model mice, a single i.p. injection of saline ( $\mathrm{n}=10)$, peramivir (20 and $60 \mathrm{mg} / \mathrm{kg}$,

$73 \mathrm{n}=10)$ was performed at $1 \mathrm{~h}$ before i.p. injection of a lethal dose of LPS (30 mg/kg) to mice, respectively. After modeling, mice survival was 74 recorded every $2 \mathrm{~h}$ until $40 \mathrm{~h}$.

\section{Preparation of the peritoneal macrophages}

76 The peritoneal macrophages were obtained from the mice after i.p. injection of $3 \mathrm{ml}$ of $3 \%$ thioglycolate as described previously. ${ }^{29}$ Briefly, the 77 mice were sacrificed, and the macrophages were isolated by lavage with $5 \mathrm{ml}$ of RPMI 1640 (Gibco Life Technologies), washed twice with PBS 
after $4 \mathrm{~h}$ of adherence, cultured in RPMI at $37^{\circ} \mathrm{C}$ and $5 \% \mathrm{CO}_{2}$, and finally stimulated with $100 \mathrm{ng} / \mathrm{ml} \mathrm{LPS}$ to harvest supernatants. The isolated

79 cells were used for cytokine analysis and cell viability assays.

\section{Preparation of the hPBMCs}

81 hPBMCs were obtained from freshly collected buffy coat fractions from healthy donors at the Tongren Hospital Affiliated to Shanghai Jiaotong

82 University, China. Briefly, hPBMCs were isolated by centrifugation over a Ficoll-Paque (Pharmacia, Uppsala, Sweden) density gradient at 800 g

83 for $20 \mathrm{~min}$ at room temperature in a Sorvall RT6000B (DuPont, Wilmington, DE, USA). Most hPBMC isolates were adherence cells that mainly

84 contained macrophages and monocytes. Isolated hPBMCs were cultured in RPMI 1640, $100 \mathrm{U} / \mathrm{mL}$ penicillin-streptomycin (Invitrogen Life

85 Technologies), and 10\% heat-inactivated fetal calf serum (Gibco Life Technologies). $3 \times 10^{5}$ cells were seeded in 96-well plates and incubated

86 for $24 \mathrm{~h}$ at $37^{\circ} \mathrm{C}$ in a humidified atmosphere containing $5 \% \mathrm{CO}_{2} . \mathrm{hPBMCs}$ were pretreated with peramivir at the concentrations of $2.5,5$ and 10

$87 \mu \mathrm{M}$ at $1 \mathrm{~h}$ before LPS $(100 \mathrm{ng} / \mathrm{ml})$ stimulation, and the supernatants were harvested at 6 or $12 \mathrm{~h}$ after LPS stimulation for cytokine analysis.

\section{Cell viability assay}

89 Cell Counting Kit-8 (TY0312, Dojindo Molecular Technology, Japan) was used to measure cell viability. Briefly, $10 \mu \mathrm{L}$ of CCK-8 solution was

90 added, and cells were incubated for $1 \mathrm{~h}$ at $37^{\circ} \mathrm{C}$. Absorbance was measured using a Cytation 5 Cell Imaging Multi-mode Reader (BioTek

91 Instruments, USA) at a wavelength of $450 \mathrm{~nm}$.

\section{Anti-inflammatory activity screening}

93 We chose an in vitro model of LPS-stimulated peritoneal macrophages to induce TNF- $\alpha$ secretion, and screened potential anti-inflammatory 
incubation of compounds at a concentration of $10 \mu \mathrm{M}$. The cell supernatant was diluted 10 -fold and the TNF- $\alpha$ content was measured with a mouse TNF- $\alpha$ Elisa kit obtained from Invitrogen. The remaining cells were subjected to CCK8 assay to detect cytotoxicity.

\section{NF-кB luciferase activity assay}

RAW264.7 cells stably transfected with an NF-kB-responsive luciferase construct, kindly provided by Prof. An Qin (Shanghai Jiaotong University, China), were seeded in 96-well plates at a density of $2 \times 10^{5}$ cells per well, as previously described. ${ }^{30}$ After $24 \mathrm{~h}$, cells were pretreated with peramivir for $1 \mathrm{~h}$ and stimulated with LPS for $6 \mathrm{~h}$. Cells were dealt with a luciferase assay system (Promega) and the luciferase activity was calculated using a Cytation 5 Cell Imaging Multi-mode Reader (BioTek Instruments, USA).

\section{Western Blotting}

Protein samples were separated by 10\% SDS-PAGE, transferred to NC membrane and blocked with 5\% non-fat milk in TBST. The membranes were washed with TBST and then incubated with the primary antibody for 6 hours at 4 degrees Celsius. The primary antibodies (1:1000) used were all from Cell Signaling Technology, USA and listed as follows: GAPDH antibody(\#2118), stat3 antibody(\#12640), phospho-stat3 antibody(\#98543), SAPK/JNK antibody(\#9252), phospho-SAPK/JNK antibody(\#4668), p65 antibody(\#4764), phospho-p65 antibody(\#3033), IKK $\alpha$ antibody(\#2682), phospho-IKK $\alpha / \beta$ antibody(\#2697), IKB $\alpha$ antibody(\#4812), phospho-IKB $\alpha$ antibody(\#2859), p38 MAPK antibody(\#8690), phospho-p38 MAPK antibody(\#4511), Erk1/2 antibody(\#4695) and phospho-Erk1/2 antibody(\#4370). Then, the membranes were incubated in HRP-linked goat anti-rabbit IgG Antibody (1:10000, Cell Signaling Technology, USA, \#7074) at room temperature for 1 hour and signals were detected by chemiluminescence (Bio rad, USA).

\section{ELISA}


TNF- $\alpha$ released by mouse peritoneal macrophages was measured by a Mouse TNF- $\alpha$ ELISA Kit (Invitrogen, BMS607-3TEN) according to the manufacturer's protocol. TNF- $\alpha$ released by hPBMCs were measured by Human TNF- $\alpha$ ELISA Kit (Youda, 1117202) according to the manufacturer's protocol.

\section{Multi-cytokine measurement}

The serum levels of a total of 12 virus-related cytokines were measured by a bead-based immunoassay panel (Mouse Anti-Virus Panel, Cat No: 740622, Biolegend, USA). The BALF levels of a total of 12 inflammatory cytokines were measured by a bead-based immunoassay panel (Mouse Inflammation Panel, Cat No: 740446, Biolegend, USA) on CytoFLEX Flow Cytometer (Beckman Coulter, USA) according to the manufacturer's protocol.

\section{Immunofluorescence staining}

Isolated peritoneal macrophages in eight-well LabTek slides (PEZGS0816, Millipore, Billerica, Massachusetts, USA) were pretreated with peramivir one hour before cell were simulated by LPS at a concentration of $1 \mu \mathrm{g} / \mathrm{ml}$ for $30 \mathrm{~min}$. Then cells were fixed in $4 \%$ paraformaldehyde, blocked with $0.4 \%$ Triton X-100/2\% bovine serum albumin at room temperature for $1 \mathrm{~h}$, and then incubated with primary antibodies for p65 (CST, \#8242, 1:400 dilution) overnight at $4{ }^{\circ} \mathrm{C}$. After being washed with PBST 3 times, the samples were incubated with Alexa Fluor 488 (Beyotime, A0423, 1:500 dilution) for $1 \mathrm{~h}$ and washed again with PBS. Nuclei were stained with DAPI. Images were obtained by confocal microscopy (TCS SP5, Leica, Solms, Germany).

\section{Histological analysis}

The left lung lobes of mice were fixed using formalin, and then the tissues were paraffin-embedded. Sections ( $5 \mu \mathrm{m})$ of formalin-fixed tissues 
were stained with haematoxylin and eosin (H\&E) according to the manufacturer's instructions, and were photographed with a microscope (Olympus Corporation, Tokyo, Japan). The histological characteristics of the lung injury (including alveolar edema and hemorrhage, the number of infiltrating leukocytes, and the thickness of the alveolar wall and epithelium) were evaluated. Each histological characteristic was evaluated on a scale of 0 to 3 ( 0 , normal; 1 , mild; 2 , moderate; 3 , severe).

\section{Statistical analysis}

Data were expressed as means \pm SEM. Statistical analyses used Student's $t$-test, two-way ANOVA or Kaplan-Meier Survival Analysis. GraphPad software was used for data analysis. Statistical significance was indicated as follows: $* P<0.05, * * P<0.01, * * * P<0.001$, n.s. not significant.

\section{RESULTS}

Peramivir is an active anti-inflammatory agent without apparent cytotoxicity.

The three neuraminidase inhibitors (peramivir, oseltamivir, and zanamivir, Fig. 1a) were explored for their ability to inhibit TNF- $\alpha$-induced by LPS in macrophages. They inhibit the elevation of TNF- $\alpha$ by $67.2 \%, 35.6 \%$ and $13.1 \%$ at $10 \mu \mathrm{M}$, respectively (Fig. 1b). Furthermore, peramivir dose-dependently inhibited TNF- $\alpha$ release with the half-maximal inhibitory concentration $\left(\mathrm{IC}_{50}\right)$ as $4.3 \mu \mathrm{M}$ (Fig. 1c). Given that the inhibitory effect of the drugs on TNF- $\alpha$ might be achieved by cytotoxicity, we tested the cytotoxicity of peramivir in macrophages by a CCK-8 assay. It was demonstrated that no apparent toxicity was observed in the peramivir-treated macrophages at concentrations up to $40 \mu \mathrm{M}$ (Fig. $1 \mathrm{~d}$ ).

\section{Peramivir inhibits LPS-induced cytokine storm in mice.}

We used LPS-induced cytokine storm syndrome (CSS) mouse model to evaluate the in vivo inflammatory inhibitory activity. Peramivir was 
administrated intraperitoneally (i.p.) at the concentration of $60 \mathrm{mg} / \mathrm{kg}$ at $1 \mathrm{~h}$ before LPS injection, and serum was collected at $4 \mathrm{~h}$ after LPS injection for further experiment. Twelve cytokines in total were simultaneously measured using a mouse antivirus panel by flow cytometric bead array. Compared with the control group, 7 cytokines including TNF- $\alpha$, IL family (IL-6, IL-1 $\beta$, IL-12), chemokines (MCP-1), interferon family (IFN- $\alpha, \gamma$ ) were significantly decreased by the treatment (Table 1 and Fig. S1). The other 5 cytokines including IL-10, IP-10, chemokine (C-C motif) ligand 5 (CCL-5), granulocyte-macrophage colony stimulating factor (GM-CSF), CXC chemokine ligand 1 (CXCL1) were slightly downregulated without statistical significance.

Given that cytokines in bronchoalveolar lavage fluid (BALF) could directly represent the inflammation status in the lungs, ${ }^{7,31}$ we examined cytokines in BALF after $8 \mathrm{~h}$ of LPS stimulation. Twelve cytokines in total were simultaneously measured using a mouse inflammation panel by flow cytometric bead array. Compared with the control group, TNF- $\alpha$ and IL- 6 were significantly decreased by the treatment (Table 2 and Fig. S2). IL-1 $\beta$, IL-10, IL-17A and MCP-1 showed downregulation without significant difference.

\section{Peramivir effectively attenuates acute lung injury and prolong the survival in LPS-induced mice.}

The histological examinations of two COVID-19 death cases both showed alveolar damage with cellular fibromyxoid exudates, pulmonary edema and interstitial mononuclear inflammatory infiltrates. ${ }^{7,31}$ The mice injected with LPS (i.p.) exhibited similar pathological features to ARDS, such as infiltration of inflammatory cells (black arrow), congestion (green arrow) and edema within thickened alveolar (yellow arrow) (Fig. 2a). In contrast, the alveolar structures of mice in the peramivir treated group were relatively intact, inflammatory cell infiltrations were significantly reduced, and mild alveolar thickening and less bleeding points or congestion were observed (Fig. 2a). Lung injury scores were calculated (Fig. 2b) to show significant protective effects of peramivir (score $=2.6 \pm 0.6$ ) to the lung tissues compared with that of control group 
(score $=4.8 \pm 0.33$ ). The survival time was prolonged in mice treated with peramivir in a dose-dependent manner after an i.p. injection of a lethal dose of LPS (30 mg/kg) compared with that in control mice (Fig. 2c).

\section{Peramivir decreases NF- $\kappa B$ transcriptional activity in RAW264.7 and the peritoneal macrophages.}

$\mathrm{NF}-\mathrm{\kappa B}$ is an important transcriptional regulator in cells that participated in inflammatory responses, of which the activation induces the expression of multiple genes and production of cytokines consequently leading to cytokine storm. ${ }^{32}$ Peramivir dose-dependently inhibited

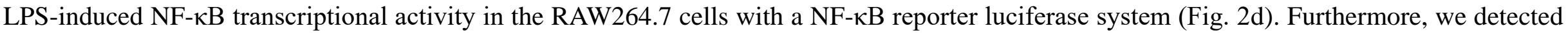
some key factors in inflammation responses by western blot and found that the LPS-induced activation of NF- $\kappa$ B pathway (phosphorylation of p65) and MAPKs (phosphorylation of p38 and Erk1/2) were inhibited after peramivir intervention (Fig. 2e). Immunofluorescence images of LPS-stimulated peritoneal macrophages treated with or without peramivir indicated that LPS-induced nuclear translocation of p65 was attenuated by peramivir (Fig. 2f).

\section{Peramivir inhibits multi-cytokine releases in LPS-induced human peripheral blood mononuclear cells (hPBMCs).}

Considering the translational value of peramivir in clinical practices, the release of TNF- $\alpha$ was tested in LPS-induced hPBMCs, which were obtained from two healthy donors. Peramivir (Fig. 3a, b and Fig. S3) significantly counteracted the level of TNF- $\alpha$ at $6 \mathrm{~h}$ and $12 \mathrm{~h}$ in a time- and dose-dependent manner without apparent toxicity.

\section{DISCUSSION}

The three neuraminidase inhibitors bear similar pharmacophoric side chains (black, Fig. 1a) and different core scaffolds (red, Fig. 1a). Peramivir 
has a five-membered cyclopentanol ring, while oseltamivir and zanamivir have six-membered ring, which might be a critical part for the anti-inflammatory activity in the chemical structure of view.

Peramivir, an intravenous neuraminidase inhibitor, was approved for the emergency use in severe influenza in 2009 by the FDA. The antiviral effect of peramivir on influenza has been described previously, ${ }^{33}$ nevertheless, few studies have paid attentions to the anti-inflammatory activity of peramivir. The activation of the immune system is attributed to the virus-induced cytokine response. ${ }^{34,35}$ Inhibition of these cytokines can potentially control the severity of the virus-induced inflammatory complications and finally reduce the mortality. ${ }^{34,} 35$ The inflammatory cytokines and influenza pathogenicity has been well correlated. ${ }^{36,37}$ In mouse model of H1N1 influenza, peramivir inhibits the levels of TNF- $\alpha$, IL6 and IFN- $\gamma$ in the lung tissue. ${ }^{38}$ And compared to oseltamivir, peramivir shows more obvious anti-inflammatory effect. ${ }^{39}$ The anti-inflammatory effect of peramivir in vivo may be due to its antiviral symptomatic treatment, while we tried to investigate whether peramivir can directly inhibit the release of cytokines by inflammatory immune cells.

As the neuraminidase is not expressed in the SARS-CoV-2 virus, oseltamivir, zanamivir and peramivir were ineffective against the virus in vitro. $^{25}$ In a clinical study, $75 \%$ of the COVID-19 patients received antiviral treatment including oseltamivir and 5 of them simultaneously infected with SARS-CoV-2 and influenza were recovered after treating with oseltamivir. ${ }^{23}$ We hypothesized that these neuraminidase inhibitors might have other effects including anti-inflammation, indicating the adjuvant therapeutic value of neuraminidase inhibitors in COIVD-19.

Viruses and bacteria induce immune cell activation and release of cytokines are Toll-like receptors (TLRs) dependent. ${ }^{40}$ The induction of

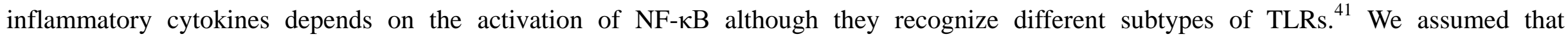

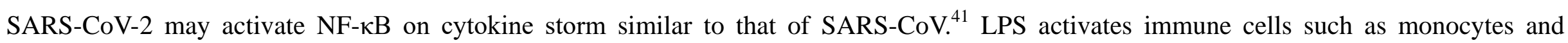


macrophages, causing the synthesis and release of inflammatory cytokines. ${ }^{28}$ TNF- $\alpha$ is one of the central cytokines involved in inflammation initiation and amplification in virus infections, ${ }^{42}$ and is reported to be elevated in critical COVID-19 cases, ${ }^{3}$ suggesting it as a proper indicator for in vitro drug screening.

Compared with oseltamivir, peramivir shows better inhibition of TNF- $\alpha$ in vitro. The reduction in TNF- $\alpha$, IL-1 $\beta$, IL-6, IL-12, IFN- $\alpha$, IFN- $\gamma$ and MCP-1 levels promoted by peramivir might play an important role in reducing stress due to the overactivated immune system and preventing organ damage of infected mice. This hypothesis was confirmed by pathological examination and lung index evaluation, which found that treatment with peramivir alleviated the severity of LPS-associated pneumonia and prolonged the survival time for mice. The lethal lung pathology caused by LPS was due to the excessive cytokine response that was primarily produced by the activated macrophages. ${ }^{28}$ Peramivir inhibited the levels of TNF- $\alpha$ and IL-6 in the BALF of LPS-induced mice. Furthermore, peramivir attenuated TNF- $\alpha$ induced by LPS both in mouse peritoneal macrophages and hPBMCs, which confirmed that peramivir can inhibit the inflammatory cytokine response mediated by macrophages.

In conclusion, the modulatory function of peramivir on LPS-induced inflammatory cytokines might contribute to the additional beneficial effect of the drug in antiviral therapy. This study provides evidence for the therapeutic value of peramivir for the potential application as an anti-inflammatory agent against cytokine dysregulation.

\section{ACKNOWLEDGEMENTS}

We thank Profs Weiheng Xu and Pei Wang from Second Military Medical University, Drs Min Liu and Weiyuan Li from Tongren Hospital 
214 Affiliated to Shanghai Jiaotong University, China for the essential assistances with this study. We thank Taozhixing biotechnology (Shanghai,

215 China) for providing us with the reagents and consumables urgently needed for the experiment. This work was supported in part by grants from

216 the National Natural Science Foundation of China (81872880, 81703506 and 81703526) and the Young Elite Scientists Sponsorship Program by

217 the China Association for Science and Technology (2017QNRC061).

218

\section{AUTHOR CONTRIBUTIONS}

L. S., C.-L. Z., W.-N. Z, Z.-B. W. conceived and designed the experiments; L. S., Y. T., D.-G. C., C.-X. Z., Z.-B. W. participated in the experiments; L. S., Y. T., Z.-B. W. analyzed the data; L. S., C.-L. Z., Z.-B. W. wrote the manuscript; all the authors provided the final approval of the manuscript.

\section{DECLARATION OF COMPETING INTEREST}

The authors declare no competing interests.

\section{REFERENCES}

[1] WHO, Coronavirus disease 2019 (COVID-19) situation report - 172. Jul 10, 2020., in, 2020.

[2] WHO. Coronavirus disease (COVID-19) Pandemic, 2020. 
[3] Huang C, Wang Y, Li X, Ren L, Zhao J, Hu Y, Zhang L, Fan G, Xu J, Gu X, Cheng Z, Yu T, Xia J, Wei Y, Wu W, Xie X, Yin W, Li H, Liu M, Xiao Y, Gao H, Guo L, Xie J, Wang G, Jiang R, Gao Z, Jin Q, Wang J, Cao B. Clinical features of patients infected with 2019 novel coronavirus in Wuhan, China, Lancet 2020;395: 497-506.

[4] Young BE, Ong SWX, Kalimuddin S, Low JG, Tan SY, Loh J, Ng OT, Marimuthu K, Ang LW, Mak TM, Lau SK, Anderson DE, Chan KS, Tan TY, Ng TY, Cui L, Said Z, Kurupatham L, Chen MI, Chan M, Vasoo S, Wang LF, Tan BH, Lin RTP, Lee VJM, Leo YS, Lye DC, Singapore Novel Coronavirus Outbreak Research T. Epidemiologic Features and Clinical Course of Patients Infected With SARS-CoV-2 in Singapore, JAMA 2020;10.1001/jama.2020.3204.

[5] Holshue ML, DeBolt C, Lindquist S, Lofy KH, Wiesman J, Bruce H, Spitters C, Ericson K, Wilkerson S, Tural A, Diaz G, Cohn A, Fox L, Patel A, Gerber SI, Kim L, Tong S, Lu X, Lindstrom S, Pallansch MA, Weldon WC, Biggs HM, Uyeki TM, Pillai SK, Washington State -nCo VCIT. First Case of 2019 Novel Coronavirus in the United States, N Engl J Med 2020;382: 929-936.

[6] Assiri A, Al-Tawfiq JA, Al-Rabeeah AA, Al-Rabiah FA, Al-Hajjar S, Al-Barrak A, Flemban H, Al-Nassir WN, Balkhy HH, Al-Hakeem RF, Makhdoom HQ, Zumla AI, Memish ZA. Epidemiological, demographic, and clinical characteristics of 47 cases of Middle East respiratory syndrome coronavirus disease from Saudi Arabia: a descriptive study, Lancet Infect Dis 2013;13: 752-761.

[7] Xu Z, Shi L, Wang Y, Zhang J, Huang L, Zhang C, Liu S, Zhao P, Liu H, Zhu L, Tai Y, Bai C, Gao T, Song J, Xia P, Dong J, Zhao J, Wang FS. Pathological findings of COVID-19 associated with acute respiratory distress syndrome, Lancet Respir Med 2020;10.1016/S2213-2600(20)30076-X.

[8] Bian X, Shi Z, Chen R, Cai J, Wang C, Xie J, Zhao L, Fei X, Zhang H, Tan Y, Zhou L, Liu Z, Ren Y, Yuan L, Zhang Y, Zhang J, Liang L, Chen X, Liu X, Wang P, Han X, Weng X, Chen Y, Yu T, Zhang X. Aveolar Macrophage Activation and Cytokine Storm in the Pathogenesis of 
Severe COVID-19, Research Square 2020;10.21203/rs.3.rs-19346/v1.

[9] Qin C, Zhou L, Hu Z, Zhang S, Yang S, Tao Y, Xie C, Ma K, Shang K, Wang W, Tian DS. Dysregulation of immune response in patients with COVID-19 in Wuhan, China, Clin Infect Dis 2020;10.1093/cid/ciaa248.

[10] Conti P, Ronconi G, Caraffa A, Gallenga CE, Ross R, Frydas I, Kritas SK. Induction of pro-inflammatory cytokines (IL-1 and IL-6) and lung inflammation by COVID-19: anti-inflammatory strategies, J Biol Regul Homeost Agents 2020;34.

[11] Mehta P, McAuley DF, Brown M, Sanchez E, Tattersall RS, Manson JJ. COVID-19: consider cytokine storm syndromes and immunosuppression, Lancet 2020;10.1016/s0140-6736(20)30628-0.

[12] Hui DSC, Zumla A. Severe Acute Respiratory Syndrome: Historical, Epidemiologic, and Clinical Features, Infect Dis Clin North Am 2019;33: 869-889.

[13] Rockx B, Baas T, Zornetzer GA, Haagmans B, Sheahan T, Frieman M, Dyer MD, Teal TH, Proll S, van den Brand J, Baric R, Katze MG. Early upregulation of acute respiratory distress syndrome-associated cytokines promotes lethal disease in an aged-mouse model of severe acute respiratory syndrome coronavirus infection, J Virol 2009;83: 7062-7074.

[14] Smits SL, de Lang A, van den Brand JM, Leijten LM, van IWF, Eijkemans MJ, van Amerongen G, Kuiken T, Andeweg AC, Osterhaus AD, Haagmans BL. Exacerbated innate host response to SARS-CoV in aged non-human primates, PLoS Pathog 2010;6: e1000756.

[15] Tisoncik JR, Korth MJ, Simmons CP, Farrar J, Martin TR, Katze MG. Into the eye of the cytokine storm, Microbiol Mol Biol Rev 2012;76: 
16-32.

[16] Channappanavar R, Perlman S. Pathogenic human coronavirus infections: causes and consequences of cytokine storm and immunopathology, Semin Immunopathol 2017;39: 529-539.

[17] Ruan Q, Yang K, Wang W, Jiang L, Song J. Clinical predictors of mortality due to COVID-19 based on an analysis of data of 150 patients from Wuhan, China, Intensive Care Med 2020;10.1007/s00134-020-05991-x.

[18] Wei H, Xu X, Tian Z, Sun R, qi Y, Zhao C, Wang D, Zheng X, Fu B, Zhou Y. Pathogenic T cells and inflammatory monocytes incite inflammatory storm in severe COVID-19 patients, Natl Sci Rev 2020;10.1093/nsr/nwaa041.

[19] Lu H. Drug treatment options for the 2019-new coronavirus (2019-nCoV), Biosci Trends 2020;14: 69-71.

[20] Li G, De Clercq E. Therapeutic options for the 2019 novel coronavirus (2019-nCoV), Nat Rev Drug Discov 2020;19: 149-150.

[21] New coronavirus pneumonia prevention and control program (7th ed.) (in Chinese). in, 2020.

[22] Richardson P, Griffin I, Tucker C, Smith D, Oechsle O, Phelan A, Stebbing J. Baricitinib as potential treatment for 2019-nCoV acute respiratory disease, Lancet 2020;395: e30-e31.

[23] Chen N, Zhou M, Dong X, Qu J, Gong F, Han Y, Qiu Y, Wang J, Liu Y, Wei Y, Xia J, Yu T, Zhang X, Zhang L. Epidemiological and clinical characteristics of 99 cases of 2019 novel coronavirus pneumonia in Wuhan, China: a descriptive study, Lancet 2020;395: 507-513. 
[24] Ding Q, Lu P, Fan Y, Xia Y, Liu M. The clinical characteristics of pneumonia patients co-infected with 2019 novel coronavirus and influenza virus in Wuhan, China, J Med Virol 2020;10.1002/jmv.25781.

[25] Li H, Wang YM, Xu JY, Cao B. [Potential antiviral therapeutics for 2019 Novel Coronavirus], Zhonghua Jie He He Hu Xi Za Zhi 2020;43: $170-172$.

[26] Lee N, Wong CK, Chan MCW, Yeung ESL, Tam WWS, Tsang OTY, Choi KW, Chan PKS, Kwok A, Lui GCY, Leung WS, Yung IMH, Wong RYK, Cheung CSK, Hui DSC. Anti-inflammatory effects of adjunctive macrolide treatment in adults hospitalized with influenza: A randomized controlled trial, Antiviral Res 2017;144: 48-56.

[27] Su L, Tu Y, Kong DP, Chen DG, Zhang CX, Zhang WN, Zhuang CL, Wang ZB. Drug repurposing of anti-infective clinical drugs: discovery of two potential anti-cytokine storm agents, Pharmcol Res 2020; Under review.

[28] Nathan C. Points of control in inflammation, Nature 2002;420: 846-852.

[29] Zhang S, Tu Y, Sun YM, Li Y, Wang RM, Cao Y, Li L, Zhang LC, Wang ZB. Swiprosin-1 deficiency impairs macrophage immune response of septic mice, JCI Insight 2018;3.

[30] Zhu S, Soutto M, Chen Z, Peng D, Romero-Gallo J, Krishna US, Belkhiri A, Washington MK, Peek R, El-Rifai W. Helicobacter pylori-induced cell death is counteracted by NF-kappaB-mediated transcription of DARPP-32, Gut 2017;66: 761-762.

[31] Luo W, Yu H, Gou J, Li X, Sun Y, Li J, Liu L. Clinical Pathology of Critical Patient with Novel Coronavirus Pneumonia (COVID-19), 
Preprints 2020: 2020020407.

[32] Chen X, Zhou L, Peng N, Yu H, Li M, Cao Z, Lin Y, Wang X, Li Q, Wang J, She Y, Zhu C, Lu M, Zhu Y, Liu S. MicroRNA-302a suppresses influenza A virus-stimulated interferon regulatory factor-5 expression and cytokine storm induction, J Biol Chem 2017;292: 21291-21303.

[33] Bantia S, Kellogg D, Parker C, Upshaw R, Ilyushina NA, Babu YS. A single intramuscular injection of neuraminidase inhibitor peramivir demonstrates antiviral activity against novel pandemic A/California/04/2009 (H1N1) influenza virus infection in mice, Antiviral Res 2011;90: 17-21.

[34] Sladkova T, Kostolansky F. The role of cytokines in the immune response to influenza A virus infection, Acta Virol 2006;50: 151-162.

[35] Tumpey TM, Basler CF, Aguilar PV, Zeng H, Solorzano A, Swayne DE, Cox NJ, Katz JM, Taubenberger JK, Palese P, Garcia-Sastre A. Characterization of the reconstructed 1918 Spanish influenza pandemic virus, Science 2005;310: 77-80.

[36] Pommerenke C, Wilk E, Srivastava B, Schulze A, Novoselova N, Geffers R, Schughart K. Global transcriptome analysis in influenza-infected mouse lungs reveals the kinetics of innate and adaptive host immune responses, PLoS One 2012;7: e41169.

[37] Tate MD, Pickett DL, van Rooijen N, Brooks AG, Reading PC. Critical role of airway macrophages in modulating disease severity during influenza virus infection of mice, J Virol 2010;84: 7569-7580.

[38] Zhang Y, Yao J, Qi X, Liu X, Lu X, Feng G. Geniposide demonstrates anti-inflammatory and antiviral activity against pandemic 
A/Jiangsu/1/2009 (H1N1) influenza virus infection in vitro and in vivo, Antivir Ther 2017;22: 599-611.

[39] Tanaka A, Nakamura S, Seki M, Iwanaga N, Kajihara T, Kitano M, Homma T, Kurihara S, Imamura Y, Miyazaki T, Izumikawa K, Kakeya H, Yanagihara K, Kohno S. The effect of intravenous peramivir, compared with oral oseltamivir, on the outcome of post-influenza pneumococcal pneumonia in mice, Antivir Ther 2015;20: 11-19.

[40] Creagh EM, O'Neill LA. TLRs, NLRs and RLRs: a trinity of pathogen sensors that co-operate in innate immunity, Trends Immunol 2006;27: 352-357.

[41] Liao QJ, Ye LB, Timani KA, Zeng YC, She YL, Ye L, Wu ZH. Activation of NF-kappaB by the full-length nucleocapsid protein of the SARS coronavirus, Acta Biochim Biophys Sin (Shanghai) 2005;37: 607-612.

[42] Malaviya R, Laskin JD, Laskin DL. Anti-TNFalpha therapy in inflammatory lung diseases, Pharmacol Ther 2017;180: 90-98. 


\section{Figure Legends}

Fig. 1 Identification of peramivir as anti-inflammatory agents. a Chemical structures of peramivir, oseltamivir and zanamivir. b Peramivir showed the strongest TNF- $\alpha$ inhibitory effect compared with oseltamivir and zanamivir. $\mathbf{c}$ The dose-response curves for the TNF- $\alpha$ inhibitions of peramivir exhibited $\mathrm{IC}_{50} \mathrm{~S}$ of $4.3 \mu \mathrm{M}$. d Cell viabilities of macrophages with peramivir treatment at different concentrations.

Fig. 2 Peramivir effectively attenuates acute lung injury and prolong the survival in LPS-induced mice. a Representative images of lung H\&E staining of control, and peramivir treatment groups. Black, green and yellow arrows indicated infiltration of inflammatory cells, congestion and edema within thickened alveolar, respectively. Scale bars, 100 or $200 \mu \mathrm{m}$ as indicated. b Lung injury scores of control and peramivir treatment groups $(\mathrm{n}=5) . * P<0.05$. c Survival time of LPS-induced CSS in control, and peramivir $(20,60 \mathrm{mg} / \mathrm{kg}$ ) groups (n=10). Kaplan-Meier analysis was performed. ${ }^{*} P<0.05,{ }^{*} P<0.01$. d RAW264.7 cells were co-cultured with peramivir at concentrations of $2.5,5$ and $10 \mu \mathrm{M}$ at $1 \mathrm{~h}$ before LPS stimulation. The activity of NF- $\mathrm{BB}$ luciferase was upregulated in all groups after $8 \mathrm{~h}$, and there was a significant decline in cells co-cultured with peramivir in a dose-dependent manner. $* * * P<0.001$. e The activation of the NF- $\kappa B$, MAPK and STAT pathway in LPS-stimulated macrophages after the treatment of peramivir. f p65 nuclear translocation in LPS-stimulated macrophages after the treatment of peramivir (blue, DAPI; green, p65; cyan, cyan). Scale bars, $10 \mu \mathrm{m}$ as indicated.

Fig. 3 Peramivir inhibits cytokine release in LPS-induced hPBMCs from a health donor. TNF- $\alpha$ concentration was elevated by LPS stimulation. $\mathbf{a}$ and $\mathbf{b}$ Peramivir reduced TNF- $\alpha$ and IL-10 release in a time (6 and $12 \mathrm{~h})$ - and dose $(2.5,5,10 \mu \mathrm{M})$-dependent manner. Peramivir showed no toxicity toward hPBMCs. $* P<0.05$, ** $P<0.01$, *** $P<0.001$.

Table 1. Clinical feature and experimental results of cytokines tested in mouse serum. 


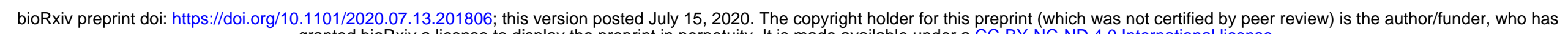
granted bioRxiv a license to display the preprint in perpetuity. It is made available under a CC-BY-NC-ND 4.0 International license.

337 Table 2. Clinical feature and experimental results of cytokines tested in mouse BALF. 
Table 1. Clinical feature and experimental results of cytokines tested in mouse serum

\begin{tabular}{ccccccc}
\hline Cytokines & Elevated in COVID-19 $^{3,49}$ & Higher in Severe Cases & & Control $(\mathbf{p g} / \mathbf{m l})$ & Peramivir $(\mathbf{p g} / \mathbf{m l})$ & $\boldsymbol{P}^{\text {Trend }}$ \\
\hline TNF- $\alpha$ & Yes & Yes & $1716.55 \pm 82.10$ & $1181.45 \pm 47.58$ & $<0.001$ & $\downarrow$ \\
IL-6 & Yes & No & $101572.3 \pm 5186.61$ & $80316.8 \pm 3464.71$ & 0.002 & $\downarrow$ \\
IFN- $\alpha$ & No Data & No Data & $649.52 \pm 17.15$ & $393.0 \pm 16.59$ & $<0.001$ & $\downarrow$ \\
IFN- $\gamma$ & Yes & No & $1521.02 \pm 154.27$ & $516.95 \pm 60.44$ & $<0.001$ & $\downarrow$ \\
IL-1 $\beta$ & Yes & No & $578.21 \pm 50.23$ & $275.12 \pm 28.97$ & $<0.001$ & $\downarrow$ \\
IL-10 & Yes & Yes & $1048.84 \pm 61.75$ & $926.39 \pm 66.67$ & 0.25 & - \\
IL-12 & No & No & $237.15 \pm 7.88$ & $119.17 \pm 7.16$ & $<0.001$ & $\downarrow$ \\
CXCL1 & No Data & No Data & $31944.11 \pm 6590.53$ & $24037.25 \pm 3011.44$ & 0.44 & - \\
IP10 & Yes & Yes & $44400.48 \pm 2953.97$ & $39370.0 \pm 1970.93$ & 0.27 & - \\
MCP-1 & Yes & Yes & $35984.98 \pm 1082.87$ & $26563.21 \pm 1257.91$ & $<0.001$ & $\downarrow$ \\
CCL-5 & No Data & No Data & $12179.03 \pm 1014.57$ & $9986.45 \pm 455.71$ & 0.19 & - \\
GM-CSF & Yes & No & $234.27 \pm 12.01$ & $190.45 \pm 9.93$ & 0.06 & - \\
\hline
\end{tabular}


Table 2. Clinical feature and experimental results of cytokines tested in mouse BALF

\begin{tabular}{ccccccc}
\hline Cytokines & Elevated in COVID-19 & Higher in Severe Cases & Control $(\mathbf{p g} / \mathbf{m l})$ & Peramivir $(\mathbf{p g} / \mathbf{m l})$ & $\boldsymbol{P}$ & Trend \\
\hline TNF- $\alpha$ & No Data & No Data & $26.39 \pm 4.53$ & $15.67 \pm 2.56$ & 0.03 & $\downarrow$ \\
IL-6 & No Data & No Data & $10176.96 \pm 2354.05$ & $3916.37 \pm 994.83$ & 0.03 & $\downarrow$ \\
IFN- $\beta$ & No Data & No Data & $30.92 \pm 5.65$ & $46.02 \pm 9.69$ & 0.23 & - \\
IFN- $\gamma$ & No Data & No Data & $67.02 \pm 20.29$ & $100.06 \pm 75.45$ & 0.35 & - \\
IL-1 $\alpha$ & No Data & No Data & $180.92 \pm 50.29$ & $186.23 \pm 53.49$ & 0.95 & - \\
IL-1 $\beta$ & No Data & No Data & $28.99 \pm 5.99$ & $22.21 \pm 2.30$ & 0.32 & - \\
IL-10 & No Data & No Data & $43.11 \pm 12.99$ & $29.82 \pm 7.20$ & 0.38 & - \\
IL-17A & No Data & No Data & $26.81 \pm 11.91$ & $17.21 \pm 4.12$ & 0.47 & - \\
IL-23 & No Data & No Data & $9.49 \pm 1.87$ & $11.45 \pm 2.85$ & 0.60 & - \\
IL-27 & No Data & No Data & $22.16 \pm 3.39$ & $20.60 \pm 4.25$ & 0.79 & - \\
MCP-1 & No Data & No Data & $900.08 \pm 285.4$ & $383.04 \pm 71.79$ & 0.12 & - \\
GM-CSF & No Data & No Data & $7.50 \pm 1.38$ & $10.08 \pm 2.17$ & 0.38 & - \\
\hline
\end{tabular}


Figure 1

a

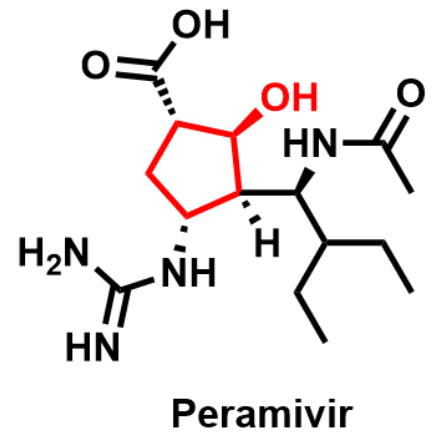

b

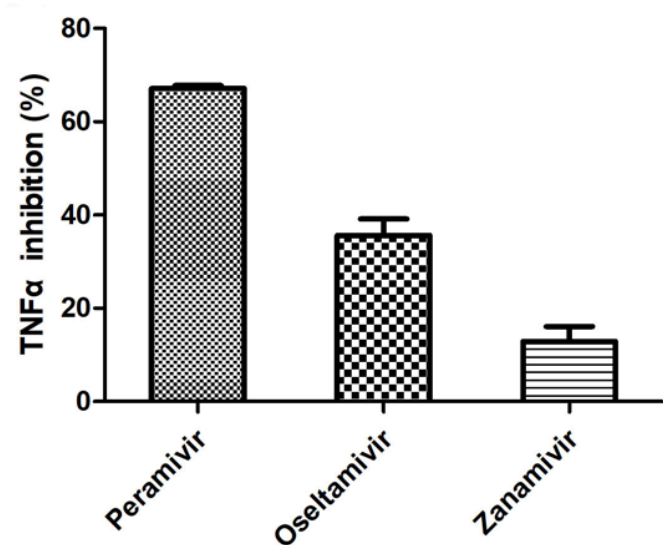

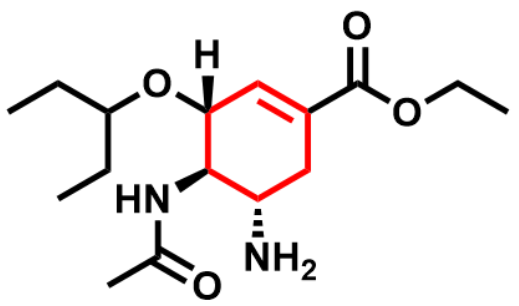

Oseltamivir

C

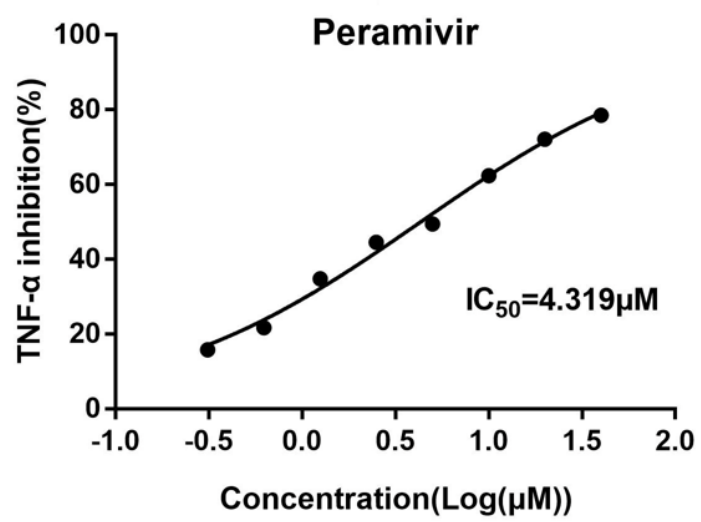

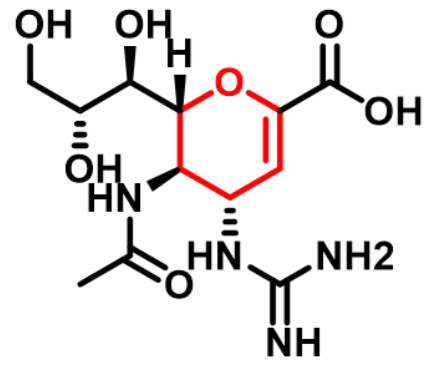

Zanamivir

d

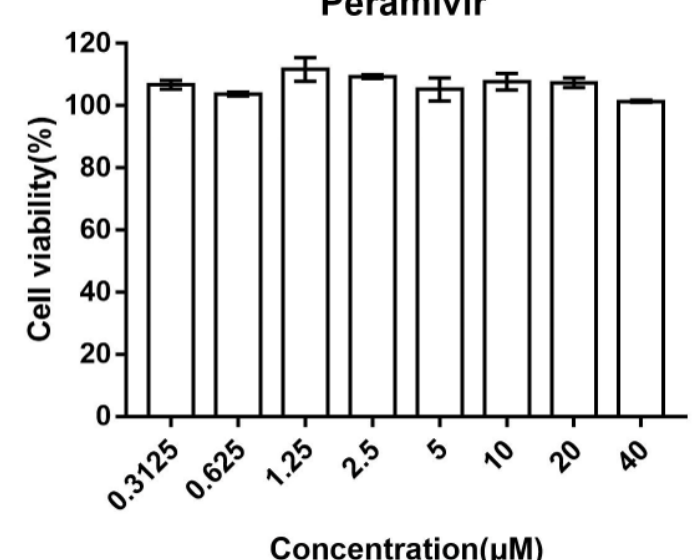


Figure 2
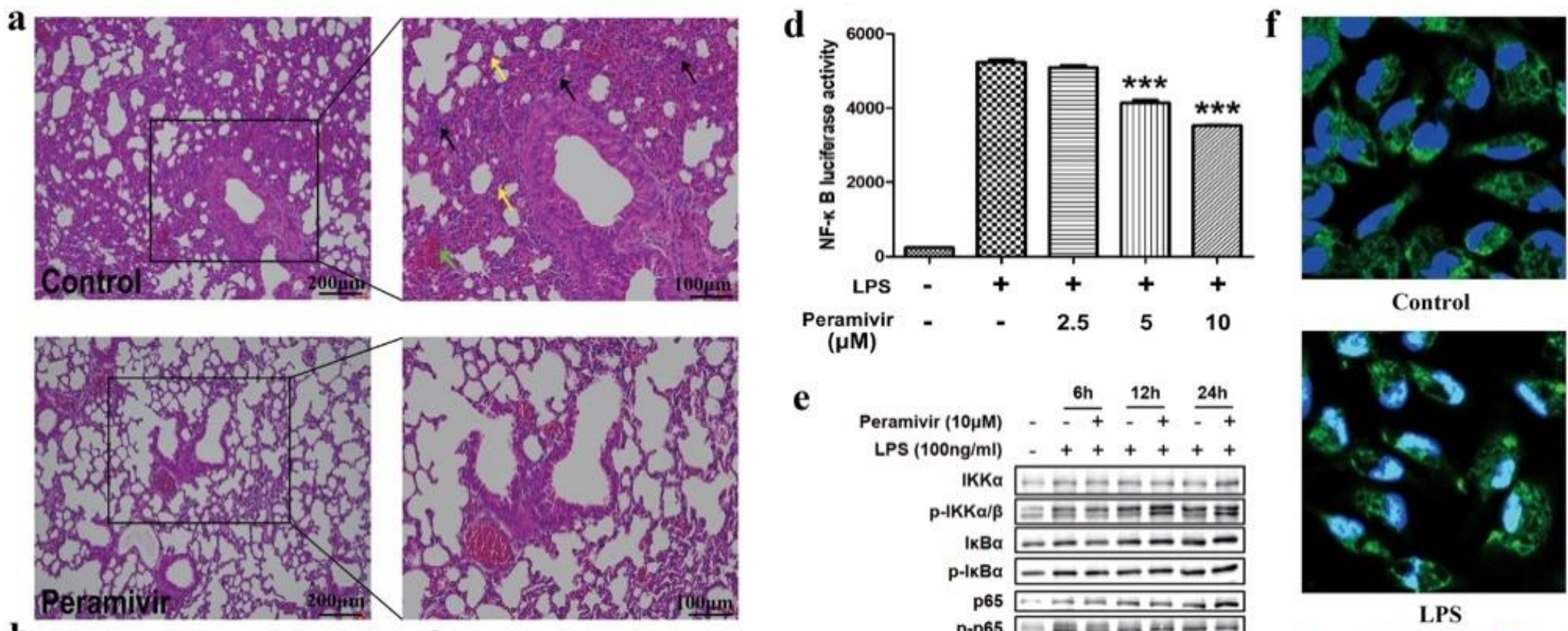
$(\mu \mathrm{M})$
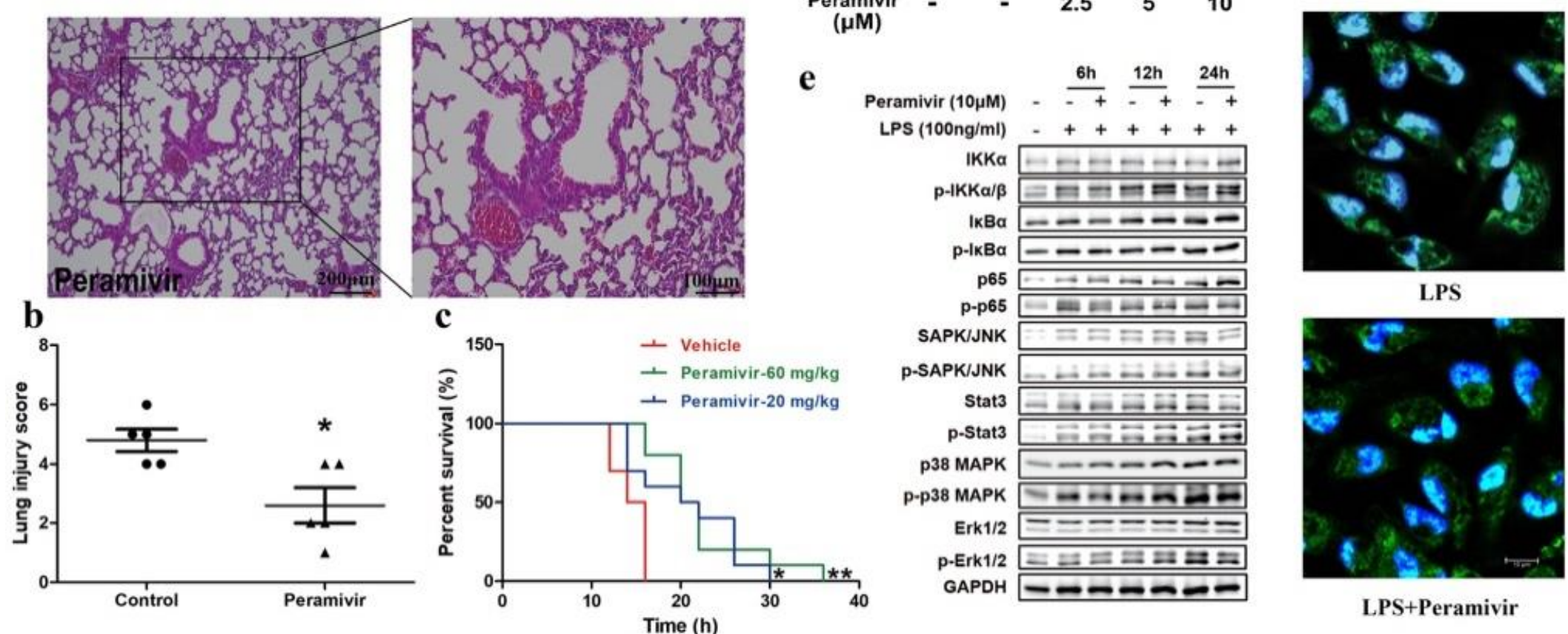

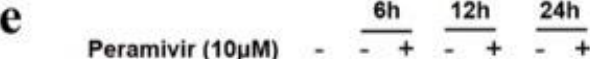 $\operatorname{LPS}(100 \mathrm{ng} / \mathrm{ml}) \quad++++++$ IKKa $-\ldots-\ldots-\cdots$ p-IKK $\alpha / \beta$ 르르료 IKBa - - - p-1кBa - -
p65 p-p65 - = SAPK/JNK $=\equiv \equiv \equiv=$

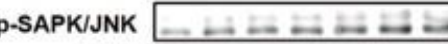
Stat3 므를
p-Stat3 프를 p38 MAPK - $-\cdots-\cdots$ p-p38 MAPK $-\cdots \cdots-2$ Erk1/2 \#=-二=三 p.Erk1/2 $E=E \equiv \equiv \equiv$ GAPDH $-\longrightarrow-\cdots-$

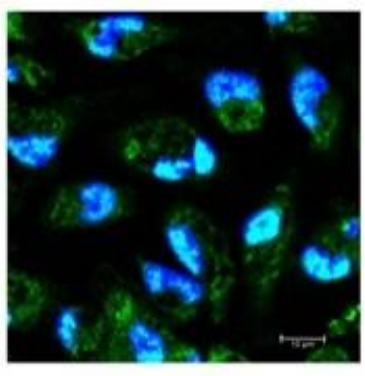

LPS+Peramivir 
$350 \quad$ Figure 3
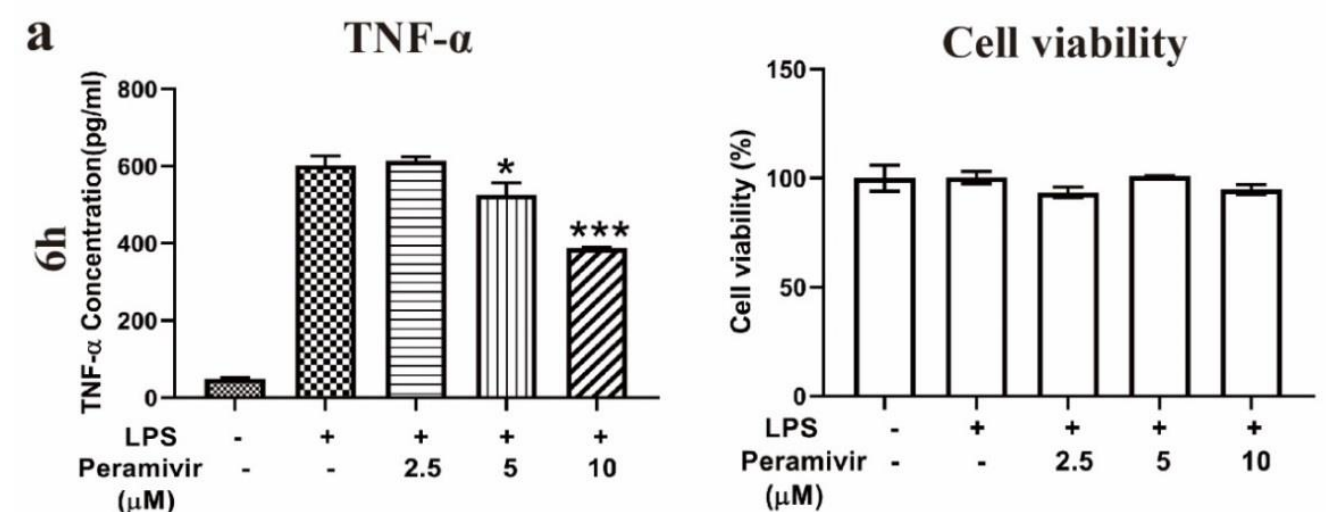

b
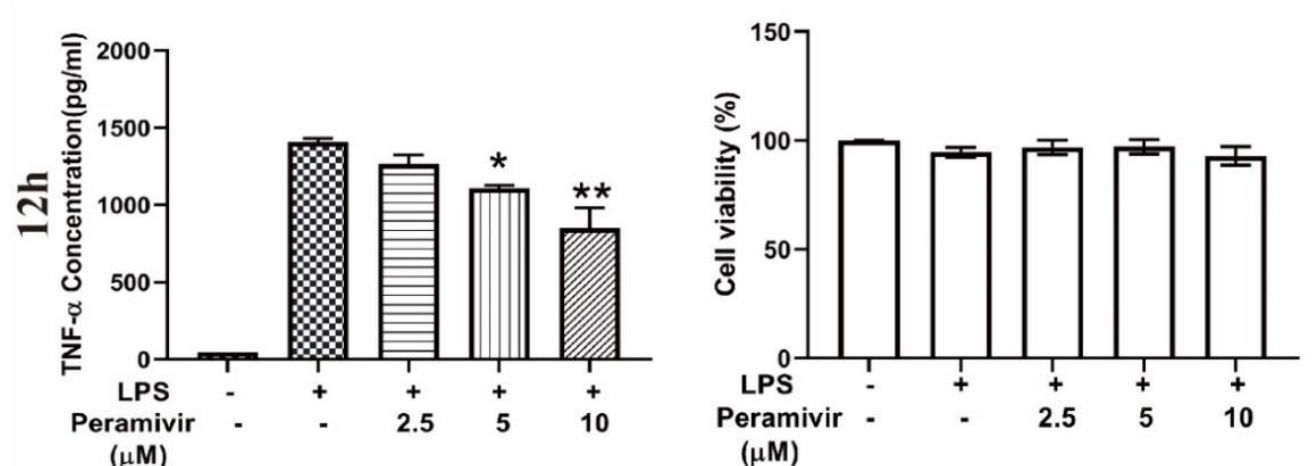

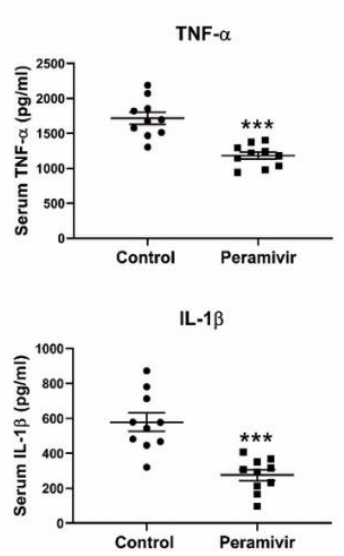

IP-10

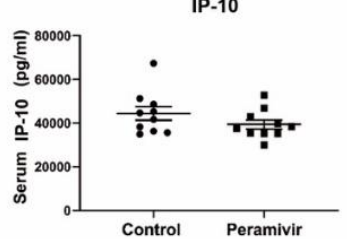

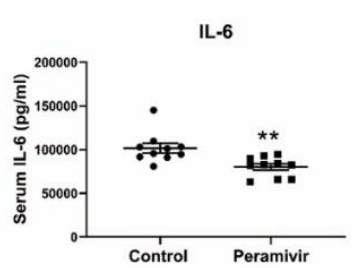

IL-10

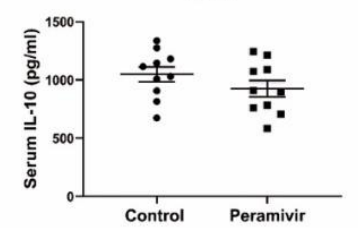

MCP-

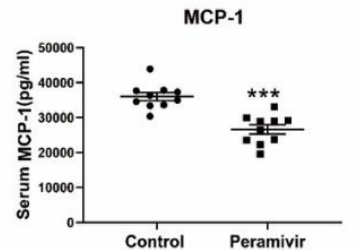

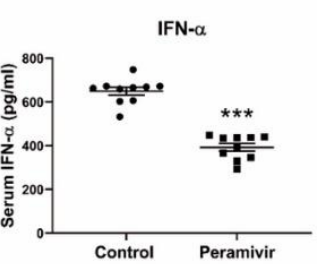

IL-12

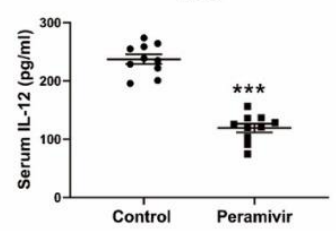

CCL-5

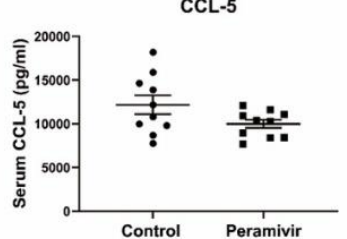

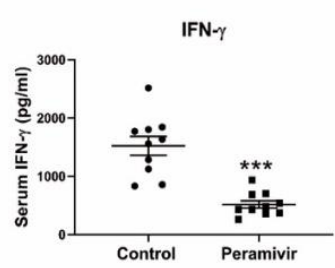

CXCL1

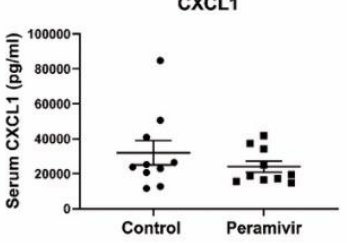

GM-CSF

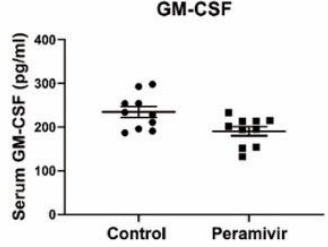

Fig. S1 Effects of peramivir on different cytokines in mice serum. Compared with the control group, TNF- $\alpha$, IL family (IL-6, IL-1 $\beta$, IL-12), chemokines 

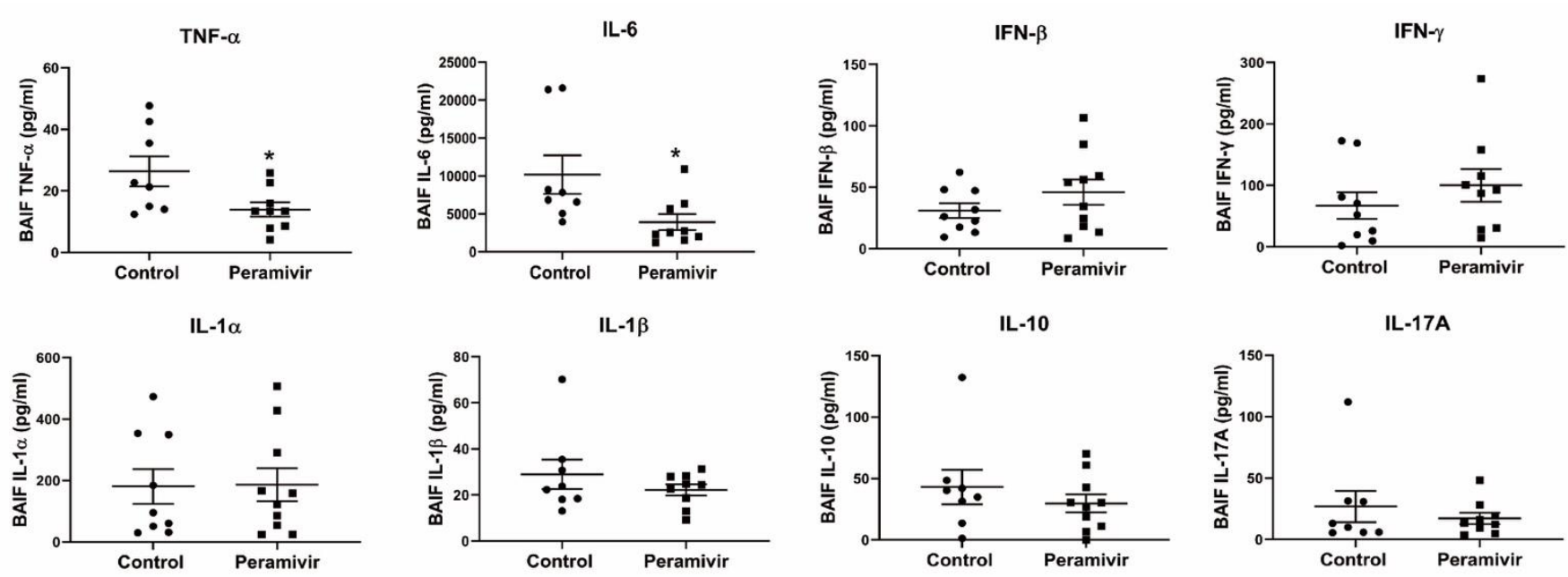

IL-23

IL-27
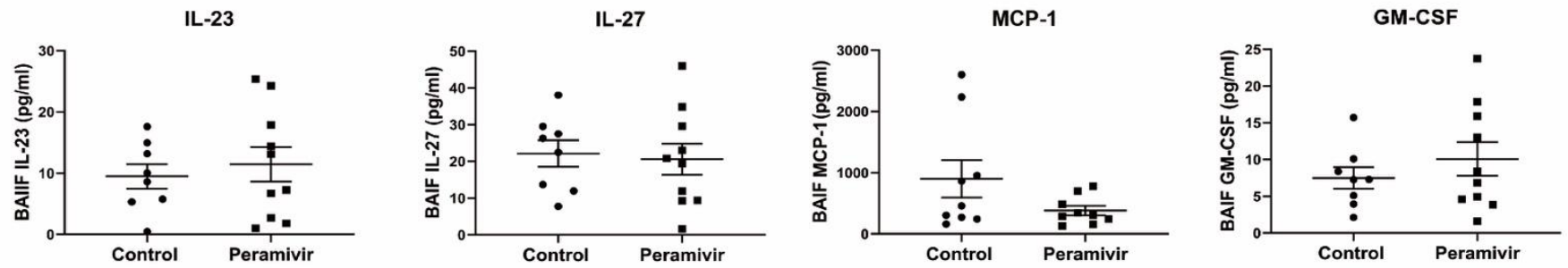

Fig. S2 Effects of peramivir on different cytokines in mouse bronchoalveolar lavage fluid (BALF). Compared with the control group, TNF- $\alpha$ and IL-6 were

significantly decreased by the treatment. $* \mathrm{P}<0.05$. 
a

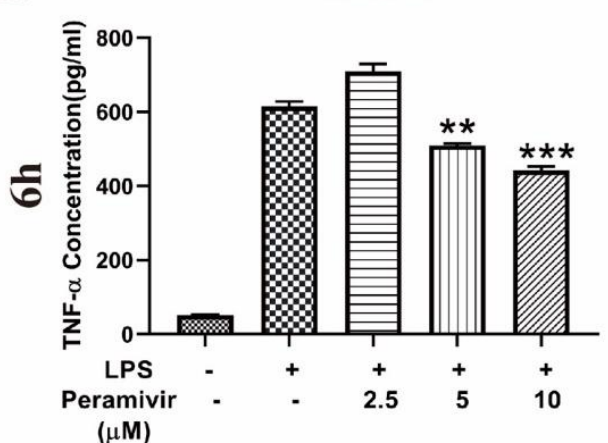

b

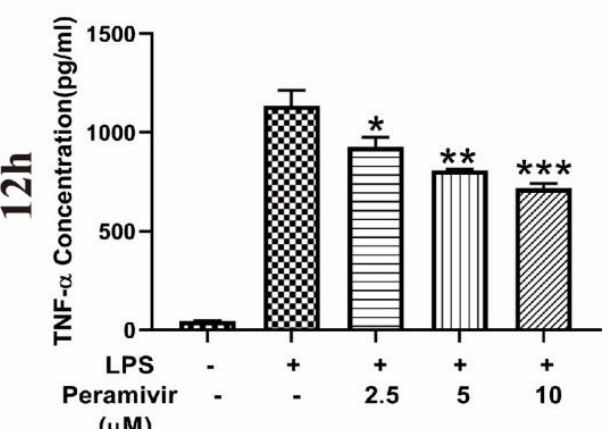

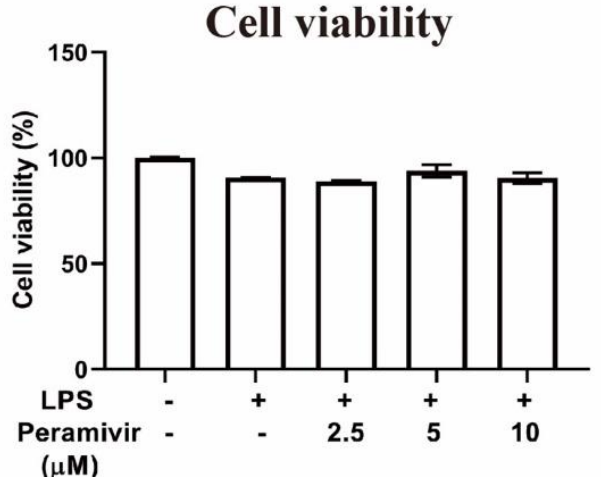

( $\mu \mathrm{M})$

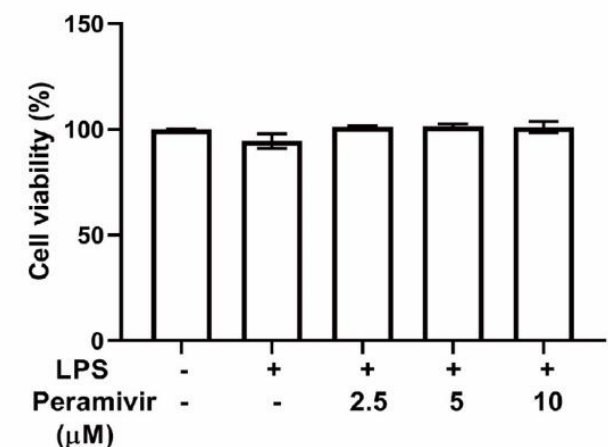

